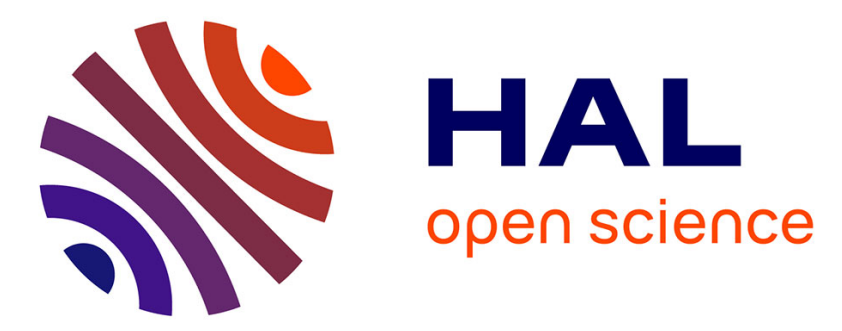

\title{
Das Lebenslange Lernen - zwischen technischer Reife, sozioökonomischer Paradoxie und kulturellem Gestammel
}

Olivier Las Vergnas

\section{- To cite this version:}

Olivier Las Vergnas. Das Lebenslange Lernen - zwischen technischer Reife, sozioökonomischer Paradoxie und kulturellem Gestammel. Klaus Künzel. Internationales Jahrbuch der Erwachsenenbildung / International Yearbook of Adult Education, Böhlau Verlag, pp.57-83, 2007, 978-3-412-37305-4. hal00690094

\section{HAL Id: hal-00690094 \\ https://hal.science/hal-00690094}

Submitted on 22 Jun 2019

HAL is a multi-disciplinary open access archive for the deposit and dissemination of scientific research documents, whether they are published or not. The documents may come from teaching and research institutions in France or abroad, or from public or private research centers.
L'archive ouverte pluridisciplinaire HAL, est destinée au dépôt et à la diffusion de documents scientifiques de niveau recherche, publiés ou non, émanant des établissements d'enseignement et de recherche français ou étrangers, des laboratoires publics ou privés. 


\section{Olivier Las Vergnas}

\section{Das Lebenslange Lernen - zwischen technischer Reife, sozio- ökonomischer Paradoxie und kulturellem Gestammel}

\section{Das Lebenslange Lernen, eine bumanistische und pragmatische Erfindung}

Seit dem Gipfel von Lissabon und der großflächigen Ausbreitung des Internet fühlt sich Europa in seiner wissensökonomischen Argumentation bestätigt, wodurch die Diskurse über die Ankunft des Lebenslangen Lernens zum Allgemeingut werden. Diese Ankündigungen beruhen in gleicher Weise auf einer humanistischen Ambition wie auf einer ökonomischen Notwendigkeit pragmatischer Art. Die humanistische Ambition ist die der promotion sociale, die bereits im Konzept der éducation permanente ${ }^{2}$ angelegt ist und von zahlreichen Autoren auf den Franzosen Condorcet zurückgeführt wird. Die ökonomische Notwendigkeit ist die der doppelten Anpassung: Zum einen jeden in die Lage zu versetzen, die vielfältigen wissenschaftlich-technischen Innovationen zu nutzen, die im Alltag in Erscheinung treten, und es zum anderen den auf Flexibilität verpflichteten Unternehmen zu ermöglichen, im Interesse der Produktivitätssteigerung von der permanenten Leistungsfähigkeit ihrer Mitarbeiter zu profitieren.

Die Beschleunigung der Innovationen hat immer auch die Weiterentwicklung der Erziehungs- und Bildungssysteme nach sich gezogen. So ist die Schulpflicht in Frankreich ab Mitte des 19. Jahrhunderts unabdingbar geworden, als offensichtlich wurde, dass die ,spontane' soziokulturelle ,Imprägnierung' der Menschen nicht mehr ausreichte, um die frühen Werkzeuge der Industrialisierung zu beherrschen. Es ist von daher nur zu verständlich, dass angesichts eines wachsenden Bedürfnisses nach Technik, das seinerseits durch die zunehmende Automatisierung der Produktion hervorgerufen wird, mit der Verabschiedung des DelorsGesetzes von 1971 der Gedanke einer allgemeinen Weiterbildungsverpflichtung eingeführt wurde. Unsere aktuelle Epoche charakterisiert sich - unter anderem durch die Überschreitung einer neuen Schwelle: die Konsequenzen der Innovationen durchdringen unser Leben mit einem viel schnelleren Rhythmus als der

\footnotetext{
la promotion sociale: der soziale Aufstieg. (siehe auch Glossar)

l'éducation permanente: siehe Glossar.
} 
Rhythmus der Generationenerneuerung, und die Weitergabe von Wissen vollzieht sich nicht mehr nur rein über die schulische und familiäre ,Imprägnierung'. Unsere Umwelt füllt sich, Jahr um Jahr, mit Objekten, die nicht spontan beherrschbar sind. Denn diese Objekte, die immer abstraktere Prozesse einleiten, werden zunehmend undurchsichtiger. Weder die, legons de choses ${ }^{3}$ der Schule noch ein gelegentliches Praktikum können dafür Sorge tragen, dass diese Objekte trotz entsprechender Beteuerungen - tatsächlich erlernt werden. Auf diese Weise drängt sich also die Notwendigkeit eines ständigen Aufholens wissenschaftlicher Vorsprünge lebenslang auf. ${ }^{4}$

Der Wille, überall die ökonomische Rentabilität zu mehren, stellt eine zweite Motivation für die Entwicklung des Lebenslangen Lernens dar: $\mathrm{Ob}$ es sich darum handelt, in einer, lean production' zu arbeiten, Strukturveränderungen bzw. Maßnahmen von Outsourcing vorzunehmen oder das Gebot der Produktionsoptimierung zu befolgen, es erscheint den Unternehmern und den Spezialisten der Personalentwicklung als immer offensichtlicher, dass eine größere Flexibilität eine höhere Produktivität sichern wird. In Anbetracht dessen nehmen die unterschiedlichen Akteure des sozialen Dialogs im Unternehmen divergierende Positionen hinsichtlich der Flexibilität ein: Manche sehen hinter diesem dehnbaren Begriff nicht die Fähigkeit der stetigen Aktualisierung oder der Richtungsänderung und Experimentierfreude, sondern eine Form der Instrumentalisierung sowie eine Polyvalenz, die mit dem Verlangen einhergeht, Personalbestände zu rationalisieren.

\section{Die Trägheit des e-learning als bezeichndendes Element des Lebenslangen Lemens}

Jedoch: Selbst wenn das Lebenslange Lernen anerkanntermaßen als ein in humanistischer Sicht und aus ökonomischen Rentabilitätsgründen unumgängliches Ziel erscheint, wie sieht es heute mit der Umsetzung in diesem Bereich aus? Wer von uns bildet sich sein ganzes Leben lang? Entsprechend der einzigen in Frankreich zugänglichen Massenuntersuchung ,formation continue 2000' der INSEEs hatten $71 \%$ der aus dem Schul- bzw. Bildungssystem ausgeschiedenen Personen unter 65 Jahren an keinerlei Weiterbildung im Jahr zuvor teilgenommen. Von

3 Begriff für die Bezeichnung naturwissenschaftlicher Fächer.

4 Die technische Evolution ist nicht die einzige, die in diese Richtung weist: Auch Entwicklungssprünge in den menschlichen Bedürfnissen und Erwartungen haben die Obsolenz gewisser Werkzeuge und Anwendungen bedingt und somit notwendige Anpassungen nach sich gezogen. Beispielsweise ist es nicht nur der Industrialisierung der Maschinen zuzuschreiben, dass das Sticken mit der Hand verschwunden ist: Um ihre einschlägigen Kenntnisse nachzuweisen, müssen junge Mädchen heute nicht mehr eine von Hand gefertigte Brautausstattung vorweisen.

5 Vgl. http://cereq.fr/cereq/fc2000/Default.htm\#Présentation sowie Céreq Bref (2001). 
diesen $71 \%$ erklärten drei Viertel, dass sie weder einen unbefriedigten Bedarf noch ein Verlangen nach Weiterbildung hätten. Anhand der gleichen Untersuchung hebt die Zeitschrift, Sciences Humaines' in einer Sonderausgabe ${ }^{6}$ über Bildung hervor, dass von den 65\% der Personen, die innerhalb der letzten zwei Jahre an keinerlei Weiterbildung teilgenommen haben, mehr als $37 \%$ aussagen, hierin keinen Nutzen zu sehen. Gewiss, 34 Prozent geben dem gegenüber an, dass sie sich gerne beteiligt hätten, man ihnen ,aber nichts angeboten hätte'. Im Übrigen ist $\mathrm{zu}$ beobachten, dass Weiterbildung immer diejenigen anzieht, die bereits am besten qualifiziert sind.

„La formation continue va aux formés. C'est là un phénomène aujourd'hui bien connu. Que l'on considère la qualification du point de vue du capital scolaire ou de la position professionnelle occupée, le constat est invariable: le taux d'accès à la formation continue des moins qualifiés est près de trois à quatre fois moindre que celui des plus qualifiés. “"

Ist also festzuhalten, dass Lebenslanges Lernen für alle lediglich ein Diskurs über Absichten ist? Als analysierendes Element dieser Frage biete ich an zu betrachten, auf welche Weise die Verbreitung der EDV-Nutzung heute einen Einfluss auf diese Frage der Kompetenzentwicklung hatte: Konnten die Wissenstechnologien im Sinne von mehr Bildung für alle eingesetzt werden?

\section{Das e-learning, noch immer ein Métier von Pionieren?}

E-learning erscheint nach wie vor als eine Angelegenheit von Pionieren. Seine Umsetzung weist starke Unterschiede auf: Ausprägungen von geradezu militantem Ausmaß finden sich neben einer vorsichtigen bzw. zweifelnden Zurückhaltung. Um diese Unterschiede zu veranschaulichen, betrachten wir den Entwicklungsstand des e-learning aus drei spezifischen Perspektiven: die Nutzung von elearning in der Hochschulbildung, seine Beziehungen zum Wissensmanagement in Unternehmen und schließlich sein Einfluss auf autodidaktische Anwendungen.

Immer mehr Universitäten sind vernetzt, und die digitalisierten Campusse und studentischen Laptops verbreiten sich zusehends. Indessen sind beide noch weit davon entfernt, ein Massenphänomen zu sein. Es existieren Pioniereinrichtungen

6 Beitrag über die Untersuchung der INSEE 2000 ist in der Sonderausgabe der Zeitschrift Sciences Humaines (2003) erschienen.

7 Bonaïti, Fleuret, Pommier \& Zomora (2006), S. 1.

Übersetzung des Zitats:

„Die formation continue erreicht hauptsächlich die Gebildeten. Dies ist ein heute allseits bekanntes Phänomen. Ob man nun die Qualifikation aus dem Blickwinkel des schulischen Kapitals betrachtet oder von der aktuellen beruflichen Position aus, die Feststellung bleibt die gleiche: Die Beteiligung der wenig Qualifizierten in formation continue ist drei- bis viermal geringer als die der besser Qualifizierten.“ 
und -kurse, die sich in diesem Bereich institutionell auf Dauer behaupten, und doch macht es noch immer keine Universität ihren Lehrkräften zur Pflicht, ihre Kurse und Übungen online zur Verfügung zu stellen. In Anbetracht der Finanzierungsprobleme hinsichtlich der PC-Ausstattung in den Mediatheken und den , cités $U^{b}$ wird es in nächster Zeit nicht von jedem Studierenden und auch nicht von jeder Lehrkraft erwartet werden können, die Kurs-Server zu nutzen.

In den Unternehmen betrachtet das Wissensmanagement seine Rolle bei der nach wie vor zögerlichen Entwicklung der Weiterbildungsintranets und anderer e-learning-Systeme als eine marginale. Von Fall zu Fall investieren die Unternehmen in Intranets, e-learning-Plattformen oder Archivierungssysteme. Im Sinne einer einheitlicheren Logik, die sich der Idee der, Lernenden Organisation' verpflichtet sieht, hätte man auf eine Integration all dieser Systeme hinwirken können. Aber tatsächlich sind derartige Strategien durch Maßnahmen verhindert worden, die jeweils eigenwilligen Interessen folgten: z.B. dokumentarische Archivierung, Pflege eines internen Kommunikationsjournals oder - alternativ hierzu einer online basierten Weitergabe von betrieblichem Know-how. Im Übrigen erweist sich eine solche Politik oft lediglich als Management der Wissensbestände, ohne größere Bedeutung für das Personal, anstatt eben ein Management durch Wissensbestände zu ermöglichen. Die Verbindung mit den Strategien vorausschauender Kompetenzplanung scheint also nicht selbstverständlich für die Verantwortlichen zu sein, die häufig nicht kompatibel mit der Logik der Personalentwicklung sind. In Anbetracht der Möglichkeiten, die wir uns von den integrierten Instrumenten des Lebenslangen Lernens erhoffen, muss dieser Tatbestand verwundern. ${ }^{\circ}$

Man hätte erwarten können, dass die durch das e-learning hervorgerufenen Möglichkeiten die autodidaktischen Anwendungen revolutioniert hätten. Stattdessen verbleibt der Traum eines Cyber-Netzwerkes des Wissensaustausches im Stadium exklusiver bzw. separatistischer Verbreitung. Die Foren sind schwierig zu identifizieren, die Beteiligten kaum zu verstehen, ihre Chats auf leidenschaftlich verfolgte Nischenthemen zugespitzt. Zugegeben, Wikipedia, diese ,kollaborative' Enzyklopädie, institutionalisiert sich zunehmend, und immer mehr Personen artikulieren sich in Blogs, aber wir sind weit davon entfernt, hier eine greifbare Massenrealität geschaffen zu haben. Gleiches lässt sich für die Methoden der

8 les cités U: Günstige Wohnmöglichkeiten für Studierende, vergleichbar mit den deutschen Studentenwohnheimen.

9 Gewiss, es gibt sie: die Beispiele einer echten Integration von e-learning und Intranet, und sie machen deutlich, dass der Begriff der ,Plattform', dem sich die netzbasierte Berufsbildung und ihre schlechte Wirtschaftlichkeit verpflichtet hat, zusehends in Frage zu stellen ist. Mit anderen Worten: Wer von einer Lemenden Organisation spricht, die mit einem distributiven ,Intranet des Wissens' ausgestattet ist, meint auch ein integriertes System der Weiterbildung und nicht eine nur zugunsten des e-leaming ausgewiesene Informationsplattform. 
persönlichen Autodidaktik behaupten: Die einschlägigen Rubriken in den Buchhandlungen weisen in diesem Bereich lediglich einige Dutzend Titel auf. Diese verteilen sich auf Methodik des Spracherwerbs, Enzyklopädien, virtuelle Museumsbesuche, schulunterstützende Methoden, die stark an Ferienhausaufgaben erinnern, sowie schließlich einige eng geführte didaktische Mittel für das, praktische Leben'.

\section{Technologische Reife, aber keine Verbreitung}

In den genannten Anwendungsbereichen des e-learning (Universitäten, Betriebe, autodidaktische Anwendungen) vollziehen sich ähnliche Entwicklungen, die von Fall zu Fall ein Re-Engineering der Lemprozesse und des Kompetenzmanagements ermöglichen. Ferner sind die Erfolgsbedingungen oft formalisiert worden, wodurch ein stabiles Niveau der technischen Reife erlangt wurde. Die Arbeiten an der Qualität des e-learning weisen darauf hin, dass verstärkt die pädagogische Relevanz verfolgt und evaluiert wird. Sobald man sich aber bemüht, den sozialen Einfluss des e-learning aus der Makroperspektive einzuschätzen, wird man eher von einem Stadium evolutionären Stotterns als von einem Zustand allgemeiner sozialer Geltung sprechen müssen. Die Frage, warum die Umsetzung der bildenden Technologien nach wie vor nicht in massiver Art und Weise in Angriff genommen worden ist, ist nicht neu. Sie kehrt seit der Erfindung der Computerlernprogramme $(E A O)^{10}$ vor 20 Jahren immer wieder. Als erste Entwicklungsbremse führte man damals den schlechten Zugang zu EDV an, und dies sowohl aufgrund der Kosten und der mangelhaften Ausstattung als auch wegen der für ihre Nutzung notwendigen Voraussetzungen. Sollten derartige Hindernisse heute noch immer Aktualität besitzen?

In diesem Feld war das letzte Jahrzehnt geprägt von einer Demokratisierung der EDV, einem verbesserten Design, sowie von einer Standardisierung der Symbole und Zeichen. Trotzdem ist der, digitale Bruch' noch nicht verschwunden. Noch vor einem Jahrzehnt wurde angenommen, dass die EDV-Nutzung der Kaste der Informatiker oder jenen raren schimärenhaften Nutzern, die auf eine doppelte Kompetenz verweisen können, vorbehalten war. Heute ist, zumindest in der Theorie, die ,individuelle' EDV-Nutzung offen für jedermann. Sollte man auf sie heute noch allergisch reagieren, wird man sich den Vorwurf gefallen lassen müssen, nicht mehr in unsere Zeit zu passen. Auf den prüfenden Blick der sozialen Norm, zunächst argwöhnisch auf die Besessenheit hochbegabter Informatiker gerichtet, folgte ein kurzer, strafender Blick auf diejenigen, die es nicht verstehen, die Früchte des Fortschritts zu ernten. So sprachen wir ehemals von einigen Insidern und heute von einigen Ausgeschlossenen. Jedoch kann der Zugang zu

10 EAO: enseignement assisté par ordinateur. Computerlernprogramme. 
EDV nicht mehr als maßgebliche Entwicklungsbarriere von e-learning betrachtet werden, auch wenn davon auszugehen ist, dass das Problem der Exklusion immer Bestand haben wird.

In der Epoche der Computerlemprogramme (EAO) bestand ein zweites Hindernis in der Zweckdienlichkeit der Werkzeuge für den Erwerb des savoir-être'1 und des Erlernens von Verhaltensmustern. Hinter dieser Fragestellung hob die Diskussion eher allgemein auf die isolierte Situation des e-Lerners ab. Was würde aus dem gemeinsamen Lernen in Gruppen werden, was aus der Koedukation oder dem situationsspezifischen Lernen sowie dem fruchtbaren Austausch innerhalb der Lemprozesse? Heute glaubt niemand mehr an eine rein technologisch aufgestellte Aus- bzw. Weiterbildung. Die Zeit gehört den ,offenen' Ausbildungen oder dem ,blended' learning. Dieser neue Ausdruck bezeichnet, vermischte Ausbildungen' oder präziser ausgedrückt: die kalkulierte Verzahnung unterrichtlicher Präsenzphasen mit Ausbildungssituationen in digital organisierter Distanz. Auch hier wiederum ist natürlich mit ein paar Lerngruppen, Foren und anderen TeleTutorien allein nicht viel erreicht. Es bleibt wahr, dass ein Missbrauch des individuellen und isolierten e-learning uns an die Vorstellung gewöhnen könnte, dass wir uns in einer primär diachronischen Kommunikationswelt bewegen.

\section{Das Lebenslange Lemen: technische Reife und potentielle Pädagogik}

Es kann also festgehalten werden, dass diese Entwicklung der Lerntechnologien es dem Weiterbildungssektor erlaubt hat, in zwei komplementären Bereichen eine gewisse technische und pädagogische Reife auszubilden. Da die Rentabilität des Lernens ausschlaggebend ist, kann das Vollstopfen des Hirns nicht das zentrale Ziel sein. Bei den Weiterbildungsakteuren ist eine doppelte Entwicklung feststellbar: zum einen eine Klärung der Rolle von Wissen und Vermittlung, wie sie unter anderem durch die Aufgabenverteilung zwischen Tutor und Designer von inhaltlichen Ressourcen bedingt ist; zum anderen die Aus- und Weiterbildungsinstitution, die nicht mehr als alleinige Inhaberin von Wissen betrachtet werden kann, da das Internet es nun ermöglicht, auf einen großen Teil nützlicher Kenntnisse Zugriff zu nehmen. In Analogie dazu beinhalten die artikulierten Weiterbildungsbedürfnisse immer häufiger Nachfragen methodologischer oder verhaltensmäßiger Art. Know-how und savoir-être gewinnen gegenüber den klassischen ,Kenntnissen' die Oberhand. Wahrscheinlich weil das Lebenslange Lernen an Erwachsene adressiert und dazu gezwungen ist, seine Rentabilität unter Beweis zu stellen, stützt es sich verstärkt auf die konstruktiven Konzepte des Wissens und des Lernens:12 Hierbei handelt es sich um Entwicklungen, die das traditionelle Machtverhältnis zwischen denen, die wissen, und denen, die sich bilden, relativieren.

11 le savoir-être: bezeichnet die Fähigkeit, an die Umwelt angepasste Aktionen und Reaktionen auszuführen.

12 siehe Carré (2005). 


\section{Neue Portabilität' des Wissens, neuer Freiraum}

Das Wissen selbst verändert seine Natur und wird sehr viel lebensdienlicher, und darüber hinaus übertragbarer, von einem Lernenden auf den anderen, von einer Situation auf die nächste: Die entwickelten Funktionen der Text- und Hypertextverarbeitung zeigen, dass die Dokumente gleichzeitig hierarchisiert, aktiv und beschreibbar sein können, und dass der Computer abstrakte Prozeduren formalisierbar und transportierbar machen kann. In diesem Sinne kann die EDV bei jedem das Verlangen hervorrufen und die Mittel liefern, sein Wissen gleich einer Skulptur zu formen, es zu validieren, um es schließlich zu vervielfältigen und auszutauschen. $\mathrm{Da}$ die zunehmende Integration des Internet in den Alltag den Bezug zu Information und Wissen verändert, kann es zudem sein, dass die hauptsächliche Umgestaltung, die das Internet dem Lebenslangen Lernen bringt, darin liegt, dass die Grenzen zwischen Informationssuche und Ausbildung, zwischen Benutzung, Memorieren und Modellierung neu gezogen werden müssen. Vielleicht wird man in einiger Zeit sagen, dass die Internetpraxis bezüglich des Wissens sowohl zu einer neuen Nähe als auch zu einem kritischen Umgang geführt hat. Gleichzeitig ist es möglich geworden, einen simultanen Zugang zum lebenslangen informellen Lernen zu organisieren. Auf diese Weise wird sich vielleicht anstelle einer sequenziellen Sicht, derzufolge sich zwischen die ,produktiven' Arbeitssituationen Phasen der Ausbildung schieben, ein neues integriertes elearning entwickeln, das einer neuen Konzeption von ,lernender Arbeit' entspricht. ${ }^{13}$ In jedem Fall haben sich an die Stelle des klassischen stagel4 mit seiner Einheit in Zeit, Ort und Teilnehmerschaft immer stärker vielgestaltige Orte personalisierter Pädagogik gesetzt. Der Lehrende erleichtert nun einen Prozess, der sich im Interesse größter Rentabilität in verteilten, frei gewählten Zeiten bzw. Orten abspielen wird. Allerdings muss klar sein, in welchem Interesse dies geschieht.

\section{Jedoch noch immer keine Lemmascbinen}

Auf diese Weise hat der technische Fortschritt den Bezug zum Wissen revolutioniert, selbst wenn diese Umwälzung nicht wirklich dort stattgefunden hat, wo sie erwartet wurde: Es sind nicht die didaktischen Verfahren, die aus den elektronischen Werkzeugen prinzipiell Nutzen gezogen haben, wenngleich sich Zugänglichkeit und Teilhabe an Wissen für gewisse Länder und soziale Milieus sehr

13 Die Konstruktion von offenen Infrastrukturen im Typus Wikipedia kann in beachtlicher Weise die Strukturierung von Netzwerken des Wissensaustausches beschleunigen. Eine wesentliche Herausforderung wäre es, das institutionalisierte e-learning mit den zahlreichen erschwinglicheren, stärker, informellen' Ressourcen zu vernetzen, wie sie das Internet vermitteln und erlebbar machen kann.

le stage: hier: Frontalunterricht als Lehrform. 
verbreitet haben. Das Internet und die Suchmaschinen haben den Zugang zum Wissen revolutioniert, und doch hat keinerlei revolutionäre ,Lernmaschine' das Tageslicht erblickt.

In diesem Bereich wird die Intelligenz der Maschinen vorwiegend genutzt, um Illustrationen zu präsentieren, Seiten zu blättern, Indices zu sortieren oder bibliographische Fußnoten zusammenzustellen. Dies geschieht allerdings selten auf der Basis eines didaktischen Urteils. Diese geringe pädagogische Nutzung der maschinellen Intelligenz verdeutlicht, dass deren Reife ihre Grenzen hat.

Im Übrigen ist dies nicht das einzige Moll, das hier anklingt: Lernende Organisation, Intranet - all diese Anwendungen existieren, ohne jedoch allgemein verbreitet zu sein.

\section{Lebenslanges Lemen: sozial-ökonomische Paradoxie}

Technische Reife sowie die Gewöhnung an EDV haben es also ermöglicht, die Anwendungsbereiche und potentiellen Klienten des e-learnings zu vermehren: Die Situation hat sich bezüglich dieser Elemente, die in den letzten Jahren die Bremsen ihrer Entwicklung darstellten, maßgeblich verändert. Nun bleibt jedoch sein sozialer Einfluss schwach: Wenige unserer Mitbürger ziehen heute ihren Nutzen aus dem e-leaming. Warum? Wenn das e-learning so wenig verbreitet ist, dann deshalb weil die erste Aufhebung der Hindernisse nicht zu seiner Verbreitung ausgereicht hat. In Ermangelung des Auffindens von ,Bremsen', muss vielleicht diese Frage der Trägheit in entgegen gesetzter Weise untersucht werden, also in Begriffen von ,Motoren'. Es reicht in der Tat nicht aus, dass eine soziale Entwicklung technisch möglich ist und nicht all zu viel Widerstand mit sich bringt, um sofort realisiert zu werden. Sie muss noch immer aktiv vorangetrieben werden.

\section{Mebr ein Problem der Motoren als eines der Bremsen}

Hier eine Fragestellung, die uns auf das sozio-ökonomische Feld führt: Welche sind die Motoren des e-learnings? Es sind die gleichen wie die des Lebenslangen Lernens im Allgemeinen - der doppelte Wille zum sozialen Aufstieg und zur Produktionserhöhung - plus ein drittes Charakteristikum der neuen Märkte, die durch die neuen Technologien geleitet werden können: Die Opportunität für Investoren Profite dank eines neuen Marktes, welcher vergleichbar mit dem von Meetic ${ }^{\mathrm{TM}}$, Amazon $^{\mathrm{TM}}$, Ebay ${ }^{\mathrm{TM}}$ oder auch Monster ${ }^{\mathrm{TM}}$ zu denken wäre, zu erwirtschaften. Versuchen wir zunächst zu verstehen, warum dieser dritte Motor nicht ausreicht: Die Produktion eines e-Ausbildungssystems hätte ebenso rentabel sein können, wie die Entwicklung von Begegnungsservices, Buchhandlungen oder Telefonangeboten. Nun aber konkretisiert sich dies heute lediglich anhand einiger DVD's oder schulunterstützender Server und einiger Internetplattformen für 
Unternehmen oder Universitäten. Dies ist wahrscheinlich bedingt durch neue Fristen und Maßstäbe der Renditen. Sie charakterisieren diesen neuen Sektor, was ihn hinsichtlich der Investitionen durch die klassischen Weiterbildungsorganismen problematisch macht, da diese nicht über ausreichendes Kapital verfügen. ${ }^{15}$

So reicht die Logik des Profits, der mit den neuen e-Produkten verbunden ist, nicht für die Verbreitung des e-learning aus: Vielmehr scheint seine geringe Inanspruchnahme darauf hinzudeuten, dass der Bedarf und damit der Markt durch Marketingspezialisten kaum werblich angeregt werden kann. Gewiss, man hätte annehmen können, dass es ausreichen würde, wenn die Spezialisten der Personalentwicklung, Arbeitgeber, andere Wirtschaftsakteure oder Sozialversicherer in e-learning investieren würden, um den Markt profitabel zu machen. Jedoch ist, sobald man über die ersten Lernphasen hinausgeht, die Motivation und die Entschlossenheit des Individuums von entscheidender Bedeutung, insbesondere im Fall des e-learning, in dem der Lernende in der Regel auf sich selbst zurückgeworfen ist. Um profitabel zu sein, braucht das e-learning ,militante Konsumenten': Ihnen müssen sich Vorteile und Mehrwert des e-leaming geradezu aufdrängen. Die wirtschaftliche Rentabilität ist abhängig von der persönlichen Investition, die jeder dem e-learning widmen kann. Die Frage nach der Glaubwürdigkeit des wirtschaftlichen ,Motors' des e-learning verweist auf das Problem der Glaubwürdigkeit der gesellschaftlichen Antriebe des Lebenslangen Lernens insgesamt. Anders ausgedrückt: Die Tatsache, dass sich keine großen Entwicklungsschübe im Bereich des e-learning verzeichnen, liegt daran, dass es Hemmnissen unterworfen ist, die weder technischer noch pädagogischer Art sind. Unserer Auffassung nach ist es der Wille zur Verbreitung des Lebenslangen Lernens, der nicht hinreichend stark ist, um das e-learning in massiver Weise zu institutionalisieren.

\section{Ein sozialer Konsens - sicherlich -, aber auch gesellschaftlich willensschwach}

Wie also verhält es sich mit den beiden Motoren, die konsequenterweise eine integrierte Entwicklung sowohl des e-learning als auch des Lebenslangen Lernens antreiben sollten: der ,humanistische Motor' auf der einen und der produktivi-

15 Das e-learning erfordert Investoren, die in der Lage sind, Infrastrukturen zu schaffen wie auch zu leiten. Es ist für die klassischen Investoren problematisch, allein diesem Wandel gerecht zu werden und neue Kategorien zu finden, vor allem nach der Verflüchtigung der Illusion der sauberen Wirtschaft. Viele erlauben es sich also zu denken, dass die wirtschaftliche Rentabilisierung nur erreichbar sein wird durch Allianzen zwischen neu auf den Markt kommenden Einrichtungen und den professionellen Anbietem der Aus/Weiterbildung, mit Produkten, die explizit eine ,Mischung' aus klassischer Weiterbildung und online-Weiterbildung wären. Jedoch muss noch herausgefunden werden, wie diese neuen Anbieter von der Bonität der künftigen Klienten überzeugt werden können. 
tätsorientierte, wirtschaftliche ,Motor' auf der anderen Seite? Um zu verstehen, warum diese beiden Motoren letztich nicht wirkungsvoller sind als der des Profits, lassen Sie uns versuchen, der Plausibilität der flankierenden Diskurse auf die Spur zu kommen.

\section{Defekte im sozialen Fabrstubl der Weiterbildung}

In Wirklichkeit ist es nicht selbstverständlich, dass die Bürger jenseits guter Absichten im Lebenslangen Lernen einen essentiellen Einsatz zugunsten ihrer persönlich gestaltbaren Zukunft erkennen. Vielleicht betrachtet sogar ein Großteil der Bürger die Verbesserung ihrer Beschäftigungsfähigkeit als Ausdruck staatlicher Gewalt, abseits eigener Verantwortung und finanzieller Mitwirkungspflicht. Einige Autoren ${ }^{16}$ sind der Auffassung, dass in Frankreich das Delors-Gesetz von 1971, das die Verpflichtung zu beruflicher Weiterbildung begründete, abweichend davon zur Vorstellung geführt hat, die formation professionnelle continue ${ }^{17}$ verkörpere eine industrielle oder neo-industrielle Zielsetzung, die dem Individuum selbst wenig Platz lässt. ${ }^{18}$ Wenn das Lebenslange Lernen wenigstens einen sozialen Aufstieg garantieren könnte, - aber nichts ist unsicherer: Erwachsenenbildung wirkt bestenfalls marginal als Instrument des Ausgleichs von sozialen Ungleichheiten. Allem Anschein nach liegt die Priorität augenscheinlich stärker auf der Ausrichtung auf individuelle Lebensläufe, in denen die Beschäftigungsfähigkeit Vorrang besitzt. ${ }^{19}$ Konsequenterweise wird das Individuum wahrscheinlich nicht erkennen können, warum es private Mittel aufwenden sollte, um eine Weiterbildung mitzutragen, die auf Kompetenzen abzielt, aus denen zweifellos wenig finanzielle Vorteile zu ziehen sind. Von vielen Unternehmen werden $V A E^{20}$ oder Weiterbildungsmaßnahmen getragen; die in diesem Zusammenhang ausgestellten Diplome und bescheinigten Kompetenzen führen aber selten zu Arbeitsplatzwechsel, Beförderung oder zu einer Gehaltserhöhung.

16 siehe im Besonderen die Arbeiten von Berton.

17 la formation professionnelle continue: (berufliche) Aus- und Weiterbildung. (siehe auch Glossar)

18 Berton (2001), S. 9.

Originalzitat:

„la formation professionnelle continue comme un dispositif industriel ou néo-industriel qui laisse peu de place à l'individu lui-même“

siehe auch Berton, Correia, Lespessailles \& Maillebouis (2004).

19 Hier im Besonderen der Text von Podevin (1999).

Zu diesem Thema siehe Dubar \& Gadéa (1999).

Siehe auch die Arbeiten der Gruppe ,Prospective des métiers et qualifications' des Generalkommissariats der Erhebung formation continue INSEE (2000) auf :

http://www.plan.gouv.fr/organisation/sas/PMQ/seance7-atel3.htm

20 VAE: la validation des acquis de l'expérience. Instrument zur Anerkennung der erworbenen Erfahrungen. (siehe auch Glossar) 
Auf der anderen Seite haben es die Arbeitgeber mit dem Problem zu tun, dass einige Langzeitinvestitionen in ihren Verantwortungsbereich fallen. Einen Arbeitnehmer auf seinen Arbeitsplatz einzustellen stellt natüllich eine Notwendigkeit dar. Jedoch ist es zu Zeiten prekärer Arbeitsverträge, in denen sowohl Arbeitgeber wie auch Arbeitnehmer mehrfach wechseln können, nicht mehr selbstverständlich, Investitionen in Weiterbildung auf lange Sicht einzugehen. Vor diesem Hintergrund erklärt sich die Entwicklung von längerfristigen Weiterbildungsgarantien, wie sie in Frankreich im congé individuel de formation (CIF $)^{21}$, der von unternehmensinternen Fonds finanziert wird, oder im droit individuel à la formation (DIF)2 , das eine finanzielle Mitwirkung des Arbeitnehmers vorsieht und auf sechs Jahre kumulierbar ist, zum Ausdruck kommen. Trotzdem erscheint die formation continue $e^{23}$ noch immer als ein Instrument, das die Qualifikationsunterschiede verstärkt, wie es C. Bonaït, A. Fleuret, P. Pommier und P. Zamora aufzeigen..$^{24}$ Man kann diesen Tatbestand im Übrigen damit in Verbindung bringen, dass die formation continue aus einer Spannung zwischen wirtschaftlichen und individuellen Motiven resultiert, Motive, deren Charakteristika durchaus in vielerlei Hinsicht gegensätzlich sind und das Lebenslange Lernen aktuell so zerrissen erscheinen lassen.

Kann die persönliche Investitionsbereitscbaft aus einem Glaubensbekenntnis heraus entsteben?

Die folgenden Feststellungen basieren auf den wenigen verfügbaren Daten zur individuellen Investition in das Lebenslange Lernen. Im Anschluss an die bereits zitierte Untersuchung ,formation continue 2000' der INSEE $^{25}$ haben von den $29 \%$ der Personen unter 65 Jahren, die das Schulsystem verlassen und an Weiterbildung im Erhebungsjahr teilgenommen haben, lediglich ein Drittel dies aufgrund individueller Initiative getan. $21 \%$ der Weiterbildungsteilnahmen wurden ausschließlich in der Freizeit der Personen getätigt, 13\% aller Teilnahmen waren selbst finanziert.

21 CIF: congé individuel de formation. Vergleichbar mit dem deutschen Bildungsurlaub. (siehe auch Glossar)

22 DIF: droit individuel à la formation. Ein durch den ANI eingerichtetes und gesetzlich verankertes Recht auf Weiterbildungsstunden. (siehe auch Glossar)

23 la formation continue: Erwachsenen- bzw. Weiterbildung. (siehe auch Glossar)

24 Vgl. Bonaïti, Fleuret, Pommier \& Zamora (2006).

25 Vgl. Revue Sciences Humaines (2003). 


\section{In kultureller Hinsicht ist das Lebenslange Lernen nicht über ein erstes Gestammel binausgekommen}

An Orten der Information und Beratung (wie die cité des métiers ${ }^{26}$ ) sind die Bürger, die freiwillig nach Weiterbildung fragen, keinesfalls mehrheitlich vertreten. Diejenigen, welche wir dort antreffen, scheinen vor allem von Notwendigkeiten getrieben zu sein. Offenbar werden ausschließlich die Bildungsangebote angenommen, die einen hohen sozialen Mehrwert versprechen (z.B. Führerschein) oder die mit einem persönlichen Mehrwert verbunden sind (in Verbindung mit sportlichen, sozio-edukativen Aktivitäten oder Hobbies und Sprachen), obwohl sie nicht, erstattet' werden. Was die Personen angeht, die selbst in beträchtlichem Umfang in eine Weiterbildung investieren, die zu 100\% außerhalb der Arbeitszeit stattfindet, so hält sich ihre Zahl in engen Grenzen: Eine solche individuelle Option scheint vor allem einige Stakhanovisten ${ }^{27}$ und ,bulimisch' Wissbegierige zu kennzeichnen sowie in einem geringeren Ausmaß Personen, die Kompetenzen in der Absicht erwerben, sich auf eine mit Sorge betrachtete Zukunft einzustellen.

Exkurs: Cité des métiers (CDM)

\section{Was ist eine Cité des métiers?}

Die Cité des métiers (CDM) sind Räume integrierter Beratung- und Ressourcennutzung und sind offen für jedes Publikum (Scbüler, Jugendliche und Envachsene), das sich auf der Suche nach Anbaltspunkten, Orientierung und Information über Berufe und das Berufsleben befindet. Ibre Mission ist es, die Nutzer mit der Realisierung möglicher Ziele vertraut zu machen. Dies gescbiebt in Form eines Angebots von drei Nutzungsvarianten: spontane Beratungsgespräcbe, gedruckte Dokumentationen und

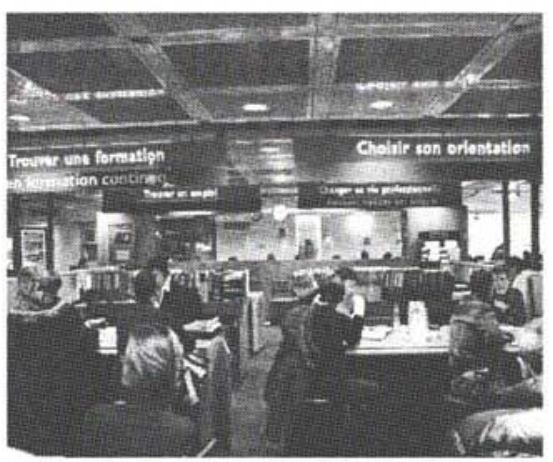

26 siehe Exkurs: Cité des métiers.

${ }^{27}$ Ausdruck geht auf den Minenarbeiter Alexeï Stakhanov zurück, der Anfang des 20. Jahrhunderts 102 Tonnen Kohle in sechs Stunden gefördert haben soll, wobei das erwartete Arbeitssoll bei sieben Tonnen lag. Die sowietische Regierung stellte diesen Arbeiter als beispielhaft hervor. Der Stakhanovismus bezeichnet also eine sowietische Doktrine, die auf einen sehr produktiven und seiner Arbeit überaus verpflichteten Arbeiter verweist. (Anm. d. Redaktion) 
freie Multimediazugänge und Ateliers, Foren und freie Informationstage. Die CDM stehen allen Zielgruppen offen, unabbängig von sozialem Status, Alter oder Bildungsniveau und sind gratis zugänglich.

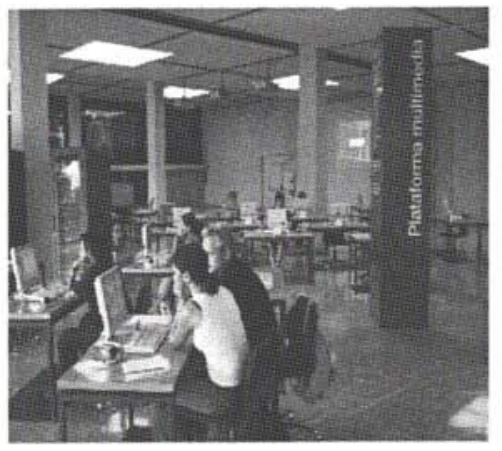

Dieses Konzept wurde 1993 geboren und bot im Rabmen der Cité des sciences et de l'industrie (CSI) von La Villette, Paris erstmalig eine Plattform, die auf diesem Nivean vielfältige Servicebereiche, ein beterogenes Publikum und Partner in einem Konzept zusammenfübrte. ${ }^{28} 6$ Jabre später eröffneten in Belfort, an der Côtes d'Armor und in Nîmes drei Einrichtungen, die durch diese Erfabrung der CSI inspiriert und geprägt wurden, Gründungen in Mailand und Genua folgten in rascher Abfolge. Seitdem entwickelt sich das Netzwerk der CDM anband einer programmatischen Vorgabe in Form eines Pflicbtenheftes" in sebr unterschiedlichen Bezirken (Metropolen wie Paris oder Barcelona, aber auch ländliche Gebiete wie l'Orne oder die Côtes d'Armor) und Kontexten (Länder wie Frankreich, die auf zablreiche Eingliederungs- und Orientierungstrukturen verweisen können neben Ländern wie Brasilien, dessen Landesinnere dergleichen Instrumente kaum aufweist.). CDM befinden sich zurzeit in vier Ländern (Frankreich, Italien, Spanien, Brasilien) sowie in 2wei weiteren Ländern (Mauritius und Portugal), wo autorisierte Projekte angelaufen sind. Ein äbnliches Konzept ist in Österreich im Rabmen der Initiative Equal ebenfalls mit Erfolg verwirklicht worden, aktuelle Projekte sind in Kanada (Britisch Kolumbien), Vietnam und Chile geplant. Die Schutzmarke Cité des métiers, die all diesen Plattformen gemeinsam ist, legt ibnen auf, ein Ort freien und kostenlosen $\mathrm{Zu}$ gangs für alle zu sein. Sie sollen für alle Aspekte und Sektoren des beruflichen Lebens offen sein und im Eingeben auf die Bedürfnisse ibrer Nutzer ibre zentrale Aufgabe seben.

Im Lebenslangen Lemen ist eine sicbtbare soziale Praxis abwesend

Um diesen Befund zu erklären, formulieren wir die Hypothese, dass die dominante kulturelle Vorstellung des Lebenslangen Lernens unfähig ist, in massiver Weise Bildungsverlangen und Nachfrage zu erzeugen. Präziser formuliert denken wir, dass drei kulturelle Hindernisse zusammentreffen, die in den entwickelten Ländern die Durchsetzung einer Gesellschaft des Lebenslangen Lernens hemmen. 
- Das erste Hemmnis verbindet sich mit der Vorstellung des ,bildenden Aktes' als einer lästigen Situation, die verknüpft ist mit den Erinnerungen an Schule und geprägt ist durch den wachsenden Spalt zwischen der Arbeitszeit und zerstreuender Freizeit.

- Das zweite Hindernis besteht darin, dass weder ein Ort, noch ein Gesprächspartner, noch ein Lernsystem existieren, von deren Existenz jedoch eine reflexive Arbeit über berufliche Zukunft abhängt, wie auch die Befähigung, Motive und Ressourcen für eine lebenslange berufliche Entwicklung zu erzeugen.

- Drittens ist auf den Mangel an Gratifikationen bzw. ,bien de salut' hinzuweisen, die von der Verwirklichung des Lebenslangen Lernens erhofft werden, und zwar in einer Gesellschaft, die allgemein durch eine starke Hierarchie des Geldes und der Bildungstitel zu charakterisieren ist.

Aus diesen drei kumulierten Effekten resultiert die Unmöglichkeit, für das Lebenslange Lernen eine lesbare Praxis, erkennbare Zeitperioden, klare Vorteile und einen universellen Referenzrahmen zu identifizieren. Dies führt dazu, dass das Lebenslange Lernen sich in keiner Weise eine sozial etablierte Existenz oder einen Ritus verleiht, den man sich einverleiben könnte. Resultiert hieraus eine blockierte Situation, die die Idee einer auf das Lebenslange Lernen fußenden Gesellschaft definitiv zum Scheitern verurteilt?

Nun ist es so, dass jedem dieser kulturellen Hindernisse ein Hebel entspricht, der zu betätigen ist, um die weitere Entwicklung voranzutreiben.

\section{Erstes Hindermis und erster Hebel:}

\section{Die Spaltung zwischen erduldeter und gewäblter Zeit überwinden}

Ob wir wollen oder nicht, aufgrund der Erinnerung an die Schule ordnen wir die Weiterbildung zwangsläufig dem Bereich der Anstrengung und der Mühsal zu. Im Gegensatz dazu bieten die Orte kultureller Bestimmung (Museen, Ausstellungszentren, Cyberspaces) ,angenehme' Situationen der Wissensaneignung. Allerdings ist festzustellen, dass diese Orte im Rahmen der ,gewählten' Zeit und in Hinsicht auf ihre Bedeutung für das Lebenslange Lernen oder das berufliche Leben nur ausnahmsweise innovative Praktiken der Erwachsenenbildung erzeugen. ${ }^{30}$ Diese Frage konfrontiert uns mit der Trennung von ,Muße' und ,Nützlichkeit'. Von einer bildenden Inanspruchnahme kultureller Orte während der Freizeit zu sprechen, scheint eine Überwindung dieser Spaltung anzudeuten, insofern Bildung im Allgemeinen mit erduldeter, kulturelle Tätigkeit mit gewählter Zeit in Verbindung gebracht wird.

30 von wenigen Bibliotheken abgesehen, siehe Bulletin des Bibliothèques de France (2002).

Der Autor stützt sich auf seine Erfahrungen in der Cité des sciences in Paris. Siehe: Las Vergnas (2006). 
Wir formulieren hier die Hypothese, dass die Öffentlichkeit nicht wahrnimmt, dass ihre Aktivitäten an diesen kulturellen Orten als Lebenslanges Lernen begriffen werden könnten, weil sie in der Mehrheit der Fälle Museen und vergleichbare Einrichtungen in der Freizeit aufsucht, welche ihrerseits zumeist nicht mit Weiterbildung in Verbindung gebracht wird, wie sie in den dominanten Vorstellungen der Arbeitswelt existiert.

Exkurs: Spaltung der Zeiten

\section{Woher kommt die Spaltung zwischen Arbeit und Freizeit?}

Historisch geseben hat sich die Spaltung zwischen diesen beiden Domänen parallel zum Auflommen der Freizeit gebildet, die ibrerseits mit der Reduzierung der Arbeitszeit (RTT) einberging." In seiner Veröffentlichung, Le sacre du temps libre: la société des 35 beures' unterstreicht Jean Viard, dass die Arbeitszeit 1948, auf das gesamte Leben geseben, noch bei 120.000 Stunden lag, wobingegen sie mit der Einfübrung der 35 Stundenwocbe in Frankeich auf 63.000 Stunden gefallen ist. Mit anderen Worten: Sie ist von $45 \%$ der damaligen Lebenszeit auf $11 \%$ der beutigen gefallen.

„De plus en plus, l'essentiel des relations sociales et personnelles se développe bors du monde du travail. La vie sociale qui, hier encore, était dominée par les rytbmes de travail, s'est progressivement structurée autour des tythmes de temps libre, de loisirs, de vacances." 32

Einige militante Vertreter der éducation populaire waren also überzeugt, dass diese gewonnene freie Zeit in den Dienst der persönlichen Entfaltung gestellt werden könne, insbesondere durch Selbstbildung und den Enverb emanzipatorischen Wissens. Die jüngsten franqösischen Untersucbungen zur Nutzung dieser, gewonnenen' Zeit zeigten aber im Gegenteil,

„que l'accroissement du temps libre ne conduit pas à une réonganisation de la structure des loisirs mais plutôt à l'intensification de pratiques existantes pour ceux qui en ont les moyens financiers.[...] Aujourd'bui, lidée selon laquelle les beures ou les joumées gagnées grâce à la RTT pourraient favoriser l'émergence d'un temps social spéafique - celui des activités, y compris de repos, choisies et pratiquées pour l'épanouissement personnel, par besoin de désaliénation, contre le professionnel et contre le domestique -, reste une utopie." 33

31 RTT: réduction du temps de travail: Reduzierung der Arbeitszeit (auf 35 Stunden), verankert im Gesetz von 2003 über Löhne, Arbeitszeit und Beschäftigungsentwicklung. Nähere Informationen (auch auf deutsch) auf http://www.35h.travail.gouv.fr/

Cazes \& Potier (2002) zitiert in Méda (2003), S. 28.

Übersetzung des Zitats:

„Mehr und mehr entwickeln sich die wesentlichen sozialen und beruflichen Beziehungen außerhalb der Arbeitswelt. Das soziale Leben, das gestern noch von Arbeitsrhythmen dominiert war, hat sich in progressiver Weise um die Rhythmen der freien Zeit, der Muße, der Urlaubszeit herum strukturiert."

33 Maresca et al. (2004), S. 69.

Übersetzung des Zitats: 
In diesem Zusammenhang ist die folgende Tabelles4 aufschlussreich:

Prozentualer Anteil der Angestellten, die aufgrund der Arbeitszeituerkürzung mebr Zeit aufbringen können für.

ausruben/schlafen

$47 \%$

sich um Familie/Kinder kümmern

$45 \%$

basteln, Gartenarbeit

$41 \%$

Familie/Freunde empfangen oder selbst besuchen

$34 \%$

shoppen

$33 \%$

TV seben

$31 \%$

Haushalt

$27 \%$

Kino-, Theater-, Restaurantbesuche

$23 \%$

Sport treiben

$20 \%$

Reisen

$16 \%$

Kreativität

$11 \%$

sich in einem Verein beteiligen

$10 \%$

Um dies $z^{u}$ verstehen, muss ein Bezug zu z2vei weiteren, simultan zur Arbeitsreduzierung auffretenden Faktoren bergestellt werden: dem machtvollen Auftritt des Weiterbildungsgesetzes souie der Entwicklung des Freizeitmarktes. Die Umsetzung des Gesetzes, das die Arbeitgeber zuingt, eine Weiterbildungsabgabe $z^{u}$ leisten, soll im Engebnis dazu geführt baben, Weiterbildungsmaßnabmen vorrangig auf Bescbäftigung und Arbeitgeberinteressen auszurichten. Fabienne Bertonss bestätigt hier, dass das französiscbe Gesetz von 1971

„a rendu la formation continue entièrement dépendante du travail (ou de son absence, ce qui revient au même). Tout ce qui n'est pas rattaché à un statut professionnel ou au domaine du travail a ainsi été évacué de la définition de la formation. Cette conception a conduit à manginaliser ou à transformer des pratiques antérieures comme l'Éducation populaire $[.$.$] , les cours d'alphabétisation ou les cours mu-$ nicipaux qui ne reposaient pas sur les mêmes prémisses. 's6

„dass der Zuwachs an freier Zeit nicht zu einer Reorganisation der Freizeitstruktur führt, aber eher zu einer Intensivierung vorhandener Tätigkeiten, was besonders für die zutrifft, die die finanziellen Möglichkeiten dazu haben. (...) Es bleibt weiterhin eine Utopie, dass die durch die RTT gewonnenen Stunden oder Tage die Entstehung einer spezifisch sozialen Zeit ermöglichen. Eine solche Zeit wäre den Aktivitäten, aber auch den Ruhephasen gewidmet, die in Abgrenzung von der Sphäre des Beruflichen und des Häuslichen vorrangig der persönlichen Entfaltung und dem Abbau von Entfremdung dienen."

CREDOC - Enquête (2002) in Maresca et al. (2004), S. 69.

Befragte: Angestellte, die die Arbeitzeitreduzierung (RTT) in Anspruch nehmen, also 26\% der Bevölkerung.

Vgl. Berton \& Correia (2004).

36 Berton \& Correia (2004), S. 28.

Übersetzung des Zitats:

"die Weiterbildung in eine vollständige Abhängigkeit von der Arbeit (oder von ihrer Abwesenheit, was auf das Gleiche hinauskommt) gebracht hat. Alles, was nicht an einem be- 
Daneben baben zablreiche Autoren, unter ibnen Jacques Demeulier auf dem CEMEAKongress in 2005, die Standardisierung und Vermarktung der Freizeit betont, die sich gleichzeitig vollzogen bat:

„Si les temps libérés peuvent être porteurs de valeurs positives, ils sont aussi porteurs d'inégalités. Ils sont un espace de marchandisation des loisirs, d'byperconsommation, de formatage des pratiques et des esprits.' 37

Hieraus folgt, dass die Idee des Lebenslangen Lemens, soweit sie sich als Erbe der Volksbildung begreift und sich einer integrierten globalen Logik der individuellen Emanzipation verpflichtet füblt, rissig wird. In Zeichen der Arbeit verschmilqt eine ,xentable' mit einer ,beschwerlichen' Weiterbildung, wäbrend sich in der Freizeit ein Markt, entfaltender' Aktivitäten per Katalogbestellung etabliert. Hier also die Aufspaltung: Auf der einen Seite die Envachsenenbildung, die zur beruflichen Weiterbildung im Sinne erduldeter Zeit geworden ist, während auf der anderen Seite die bezablten Urlaubstage vom Marketing und der Freizeitgesellschaft absorbiert werden. Hierbei spricht man von gewählter Zeit - gewäblt, aber vermarktet. Die Selbstbildung, jener bistorische Motor des individuellen sozialen Aufstiegs, wird im Zuge eines solchen Prozesses ausgelöscht. Die kulturellen Produzenten baben angesichts des überwältigenden Einflusses der Quotenrechner keine andere Wabl als sich in Marketingprofis zu verwandeln, die ein Freizeitverständnis favorisieren, das auf die Ziele der Entspannung und Unterhaltung hin kalibriert wird.38

Indessen wäre es grob vereinfachend zu behaupten, dass die Spaltung der Zeiten allein dafür verantwortlich zu machen sei, dass sich an kulturellen Orten keine Praktiken selbstgesteuerten Lebenslangen Lernens durchsetzen konnten. ${ }^{39}$ Als

ruflichen Status bzw. an der Arbeitswelt festmacht, wurde so aus der Definition der Weiterbildung ausgeschlossen. Diese Entwicklung hat dazu geführt, dass herkömmliche Bildungspraktiken, die nicht auf den nun durchgesetzten Prämissen beruhten, wie beispielsweise die éducation populaire, Alphabetisierungskurse oder kommunale Grundbildungsangebote, marginalisiert oder ,angepasst' wurden [...]."

37 Demeulier (2005), S. 5.

Übersetzung des Zitats:

„Wenn die nun geschaffene Freizeit Träger positiver Werte sein kann, ist sie auch Träger von Ungleichheiten. Sie ist ein Raum der Vermarktung, des Hyperkonsums, der Formatierung von Handlungen und des Geistes."

38 Wir beziehen uns hier auf die „3 D“, die nach J. Dumazedier (1962), S. 26f. drei komplementäre Dimensionen der Freizeit definieren: Délassement, Divertissement et libre Développement. (= Entspannung, Zerstreuung und freie Entwicklung). Siehe z.B.:

http://www.culture.gouv.fr/culture/dp/mpe/recherche/pdf/ET_55.pdf;

ein kurzer Abriss über sein Leben und Werk findet sich unter:

http://www.peuple-et-culture.org/IMG/pdf/doc-97.pdf

39 Hierfür sind drei komplementäre Gründe heranzuziehen. Auf der einen Seite befinden sich diese Orte außerhalb der traditionellen Wirtschaft und der renditeorienterten institutionellen Weiterbildung. Auf der anderen Seite stehen die kulturellen Freizeiteinrichtungen den 
sicher gilt, dass diese Spaltung heute das Konzept eines integrierten Lebenslangen Lernen verschwimmen lässt, indem diese beiden Amalgame: gewählte Zeit = Freizeit + berufliche Nutzlosigkeit einerseits und erduldete Zeit $=$ Arbeit + berufliche Bildung andererseits gegenübergestellt werden. Ganz im Gegensatz hierzu muss man das Verständnis der Idee, emanzipatorischen Wissens' fördern, die diesen Trennungsschritt transzendiert. Dies sollte zu einer angemessenen Würdigung des Lebenslangen Lernens führen und es ermöglichen, gegen das Image der Beschwerlichkeit anzukämpfen, das tendenziell mit jeder bildenden Tätigkeit assoziiert wird, die mit der Arbeitswelt zusammenhängt. Gleichzeitig gilt es, gegen die Vorstellungen von Belanglosigkeit anzugehen, die mit Freizeitaktivitäten verknüpft werden könnten.

\section{Zweites Hindernis und zpeiter Hebel: \\ Die kulturelle Revolution des Lebenslangen Lemens deutlich zum Ausdruck bringen}

Das Lebenslange Lernen ist vor allem eine kulturelle Revolution, die sich aber noch nicht vollzogen hat. Es antwortet auf neue kulturelle Bedürfnisse, die sich bestätigen werden, weil sich die Gesellschaft sowohl in ihrem Bedürfnis nach Wissen wie hinsichtlich des Raums, den sie der Arbeit zubilligt, gewandelt hat. Allerdings sind diese Bedürfnisse noch nicht offenkundig. Bis jetzt ist das Lebenslange Lemen lediglich ein diffuses Agglomerat widerstreitender Elemente. Sie müssen lesbar und sichtbar gemacht werden.

Exkurs: Lebenslange Orientierung

\section{Warum von lebenslanger Orientierung sprechen?}

Wir erleben einen Orts- und Sinneswandel von Arbeit und Beruf, der die Basis unseres kulturellen Systems maßgeblich verändert. Der Beruf, der bis beute das Wesentliche unserer Identität ausmacht, kann beute $2^{4}$ einem ,temporären Beschäftigungszustand' werden; die Arbeit dematerialisiert und delokealisiert sich und spannt sich über die Zeit. An die Stelle des Arbeitsvertrages scheint sich der Aktivitätsvertrag setzen zu können, der eher das Produkt als die Arbeitskraft zum Gegenstand bat. Was die Beqablung angebt, könnte diese der utopiscbsten Annabme zufolge in einigen Jabrzebnten nicht mebr als Entlobnung für

schulischen Ausbildungsstätten zu fern, den Orten der Muße hingegen zu nah: Tatsächlich formatieren die Betreiber des kulturellen Marketings ihre Inhalte in konsumierbare und freizeitorientierte Objekte. Und schließlich bieten sie nur wenige Möglichkeiten, Kenntnisse zu mobilisieren, um diese in integriertes Wissen zu transformieren. Die meisten der öffentichen Netzwerke in Frankreich wie die CIO, ALE, AFPA und CARIF lassen sich durch folgende Ambiguität charakterisieren: Sie wünschten häufig, Herrn oder Frau Jedermann direkt zu informieren oder zu beraten, besitzen dafür aber kein offizielles Mandat. 
eine ,Mübsal', sondern als Sicherung des Lebensunterbalts verstanden werden. Dies sind zablreiche Entwicklungen, die den Gedanken nabe legen, dass der Arbeitsplatz als zentraler Bezugspunkt des individuellen und kollektiven Gleichgewichts in Frage gestellt wird.

In gleicher Weise zeigt das Verschwinden des impliziten Systems, beruflichen Erfolges' die Wichtigkeeit einer professionellen Orientierungsarbeit für alle auf. In den entwickelten Länderm sind berufliche Laufbabnen nur noch selten Resultat der Forffübrung einer Familientradition. Noch vor 10 Jabren arbeitete in Frankreich der Sobn eines Eisenbabners oft bei der Babn. Wenigstens eines der Kinder übernabm den Handel bow. den landwirtschaftlichen Betrieb der Familie. Obne Zweifel bestand das entscbeidende Kriterium im beruflichen Erfolg. Man boffte, es über den $W$ eg des sozialen Aufstiegs besser als die Eltern $z^{u}$ machen. Aber im Wesentlichen war der Reiseplan durch den Kontext, die Ressourcen und das Familienmodell vorgegeben. Der Anteil freier Wabl war begrenzt und die Information über das Berufsleben verlief über die ,familiäre Imprägnierung'. Die Möglichkeiten des sozzialen Aufstieges waren selten, bauten auf Freiwilligkeit und Bebarrlicbleeit auf und waren relativ deutlich abgesteckt: Man lebte sein Leben im gleichen Unternebmen, mancbmal in der gleichen Anstellung; die Berufe waren vielfältig, leicht $z^{u}$ benennen und $z^{u}$ beberrschen und mebrbeitlich auch gut zugänglich, da sie oft mit Gesten, Werkzeugen, Maschinen oder Lebensarten verknüpft waren. Diese quasi vererbte, vorbestimmte Orientierung generierte also wenig Bedarf nach Information und Beratung binsichtlich der Berufswabl oder der Entwicklung von Lebenszielen. Da Begriffe wie ,Projekt' oder ,Laufbabn' also nur begreñten sozialen Nutzen versprachen, traten sie nur wenig bervor. Aus diesem Grund sind die Fragen, die das Lebenslange Lemen heute stellt, auch kultureller Natur. Was das Lebenslange Lemen progressiv erscheinen lässt, sind die Wandlungen in den Repräsentationsformen von Arbeit, Beruf, Erziebung und in einer globaleren Sicbt von Identität und schließlich von einer Investition in die Zukunft: Es handelt sich fortan darum, nicht mebr lediglich in $\mathrm{Be}$ grifflicbkeiten der Erstausbildung, sondern in denen des Lebenslangen Lernens $z^{u}$ denken. Konsequenterweise sind dabei die zablreicben bildenden Situationen zu bedenken, anzuleiten und Gewinn bringend zu nutzen und ein ,berufliches Projekt' auszudenken und $z^{u}$ entwickeln.

Falls das Verschwinden der beruflichen Imprägnierung durch die Familie gleichzeitig Offenbeit und Unsicberbeiten erzeugt, wie soll verbindert werden, dass sich dies in Friktionen, Orientierungslosigkeit und Dequalifizierung auswirkt? Was verleibt jedem den Willen und die Mittel, im Rabmen des Möglichen Autor seines beruflichen Lebens zu werden? Notwendig ist ein Höchstmaß an Möglichkeiten, die jedem eingeräumt werden sollten, um von den verfügbaren Handlungsspielräumen zu profitieren. So kann der Bereich potentieller Freibeit in den Dienst des freien Willens gestellt werden. Darin liegt eine conditio sine qua non für den Erfolg der Kernziele des Lebenslangen Lermens, nämlich die Validierung erworbener Kompetenzen, die freinillige Bilanzierung von Kompetenzen oder der Bildungsurlaub (CIF) brv. das individuelle Recht auf Bildung (DIF). Die Notwendigkeit eine Kultur der Berufsorientierung für jedes Alter zu begrïnden erstreckt sich nicht nur auf die Kenntnis der Arbeits- und Berufswelt, sonderm auch auf Selbstbildung und Selbstkenntnis. 
Das Mögliche kennen, daraus wäblen zu können sowie sich eigener Grenzen und Werte bewusst zu sein, darin sind Kompetenzen $z^{u}$ seben, die untrennbar mit jeder gewäblten Orientierung Gültigkeit beanspruchen. In ibnen erschließt sich die Autonomie des Individuums.

Nun fällt es zugestandenermaßen leicht, innerhalb der Orte und Dienstleistungen, die sich den individuellen Bedürfnissen glaubhaft verpflichtet fühlen, Diskrepanzen festzustellen. Wie im Falle des e-learning sind die jedermann zugänglichen Informationen und Beratungsangebote Sache einiger weniger Pioniereinrichtungen. Natürlich gibt es zahlreiche andere Lernorte, sie sind aber immer nur einer bestimmten Zielgruppe gewidmet: Sie sind aufgrund öffentlicher Vorschriften auf ein spezifisches Publikum oder auf die Beförderung eines bestimmten Ziels ausgerichtet und finden sich dementsprechend eher in der Logik des Einübens als in der des Motivierens wieder. So muss man sich dem Offenkundigen beugen: Trotz der Bemühungen der militanten Befürworter der Erwachsenenbildung und promotion sociale verkörpert das Lebenslange Lernen noch ein vorgeschriebenes Verhaltensgebot, das jedoch von den Individuen nicht im Sinne einer Möglichkeit gelebt wird, sich selbst zu organisieren und ihre berufliche Zukunft zu bestimmen. Nun kann aber aus den Ergebnissen der Studie, formation professionelle' der INSEE 2000 mit Blick auf die Beteiligungsbarrieren zu Weiterbildung abgeleitet werden, dass deren Beseitigung eine Vielzahl von entsprechenden Vorschlägen, Möglichkeiten und Rechten voraussetzt.40

\section{Drittes Hindermis und dritter Hebel: \\ Die Validierung der envorbenen Kompetenzen vergrößerm, um bieraus soziale Fabrstublef- fekte zu errielen}

Die Diplome bestimmen die sozioökonomische Position eines Jeden und man verschafft sie sich, wenn man jung ist. Danach sind Bildungsabstände nie wieder aufholbar! Dies entspricht jedenfalls einer von der Mehrheit geteilten Vorstellung. Nun bedeutet aber die Verteidigung des Lebenslangen Lernens auch, jene

40 Eine Untersuchung der DARES (2001), zitiert durch F. Berton, op.cit,, weist übrigens darauf hin, dass "man innerhalb der Unternehmen, die sich am wenigsten für die Weiterbildung ihrer Arbeitnehmer engagieren, auch Bildungsurlaub am seltensten nachgefragt wird, während andererseits die Arbeitnehmer der, weiterbildungsintensiven' Unternehmen den Bildungsurlaub am meisten nutzen. [... Dies legt die Vermutung nah, dass dafür die genauere Kenntnis der Weiterbildungsziele seitens der entsprechend sensibilisierten Arbeitnehmer verantwortlich ist."

Originalzitat:

„c'est dans les entreprises qui forment le moins leurs salariés que l'on trouve aussi le moins de demande de congé et ce sont les salariés des entreprises particulièrement impliquées dans la formation qui en bénéficient le plus [... ce qui peut laisser penser que cela vient d'une] meilleure connaissance des dispositifs de formation de la part des salariés déjà sensibilisés à la formation." 
Vorstellung zu relativieren. So denken einige Autoren, dass die Einführung und Verbreitung der $V A E$ es ermöglichen wird, unsere Vorstellung vom Lebenslangen Lernen zu beflügeln.

Sandra Bellier ${ }^{41}$ beispielsweise führt in ihrer Veröffentlichung ,Le e-learning, pédagogie, contenus, modalités, acteurs.' aus:

„Le e-learning est un moyen puissant de faire évoluer les comportements individuels vis-àvis de la formation. (...) les systèmes d'évaluation et de reconnaissance des acquis deviennent indissociables du e-leanning. $\mathrm{Si} \mathrm{je} \mathrm{me} \mathrm{forme} \mathrm{individuellement,} \mathrm{je} \mathrm{dois} \mathrm{toujours}$ avoir accès aux moyens d'évaluer et de faire reconnaître mes progrès. Et cette reconnaissance doit en outre être socialement utile...."

Aber wird dies die soziale Nachfrage nach Lebenslangem Lernen hinreichend verstärken können? Die Möglichkeit, individuelle Erfahrungen anerkennen und akkreditieren zu lassen, ist in Frankreich seit 1985 gesetzlich geregelt und wurde 1992 erweitert, ohne damit die Nachfrage nach Selbstbildung wesentlich zu steigern. Nichts deutet also darauf hin, dass die vielfältigen Möglichkeiten, eine Anerkennung in Form von Diplomen oder professioneller Kompetenzzertifikate zu erhalten, die Zahl der Individuen, die bereit sind, in ihre Kompetenzentwicklung zu investieren, signifikant erhöht hätte.

Exkurs: Validation des acquis de l'expérience (VAE)

\section{Welche Aufgabe hat die VAE in rechtlicher Hinsicht?}

Die VAE ermöglicht es allen Personen, die seit mindestens drei Jabren im aktiven Berufsleben stehen, ibre beruflichen Kompetenzen in Gestalt eines Titels, eines Diploms beruflicher Ausricbtung oder eines Qualifikationszertifikates offiziell anerkennen $z^{u}$ lassen. Die $V A E$ wurde am 17. Januar 2002 durch das Gesetz über die modemisation sociales ins Leben gerufen.

41 Vgl. Bellier (2001).

42 Bellier (2001), S. 102.

Übersetzung des Zitats:

„Das e-learning ist ein machtvolles Mittel, um individuelle Verhaltensweisen angesichts der Weiterbildung zu entwickeln. (...) Die Evaluations- und Kompetenzanerkennungssysteme sind nun untrennbar mit dem e-leaming verbunden. Wenn ich mich individuell weiterbilde, muss ich immer Zugang zu Evaluierungsinstrumenten und zu Möglichkeiten haben, meine Fortschritte anerkennen zu lassen. Und diese Anerkennung muss zusätzlich noch sozial nützlich sein..."

43 la loi de modernisation sociale du 17 janvier 2002. Das Gesetz vom 17. Januar 2002 zur sozialen Modernisierung Frankreichs kann im Internet eingesehen werden auf: http://www.legifrance.gouv.fr/WAspad/UnTexteDeJorfnumjo=MESX0000077L. 
Die $z^{u}$ validierende Erfabrung muss inbaltlich mit dem beantragten Abscbluss übereinstimmen und kann im Zuge bezablter, unbezablter oder ebrenamtlicher Tätigkeiten enworben worden sein. Das Diplom kann vollständig auf diese Weise erlangt werden, d.h. obne dass eine Weiterbildungsteilnahme vorliegt oder Prüfungen absolviert werden müssen. Für den Fall, dass die Jury der VAE eine Zertifizierung nur in Teilen vornehmen kann, ist es möglich, die nicht akkereditierten Einbeiten durch eine neue Phase beruflicher Erfabrungen oder durcb Weiterbildung auszugleichen. Die Akkereditierung erfasst alle beruflicben Titel, Diplome und Qualifizierungsnachweise. Es bleibt binzuzufuigen, dass darin auch die beruflichen Abschlüsse entbalten sind, die im Rabmen des neuen Repertoires beruflicher Qualifikationen (RNCP)" auftauchen.

Die Inanspruchnabme der VAE ist nicht kostenlos. Die Aufvendungen sind variabel (durcbscbnittlich 100€), je nach Ministerium und beantragtem Diplom. Eine Übernabme der Kosten kann einem Arbeitnebmer des privaten Sektors durch eine Fondgesellschaft bewilligt werden, wobei darin sowobl die Aufwendungen für sein Gehalt, als auch die Kosten für die VAE selbst eingeschlossen sind. Es existiert ein Rechtsanspruch auf Beurlaubung zum Zwecke der VAE. Dieses siebt einen Sonderurlaub von bis zu 24 Arbeitsstunden vor, um sich der VAE-Jury vorzustellen und evtl. um sich bei der Erstellung der Antragsunterlagen unterstützen zu lassen. Die VAE wird in juristischer Hinsicbt der Weiterbildung zugerechnet. Für den Fall, dass die angestrebte Zertifizierung im Repertoire der Berufsbildungsabschliusse auftaucbt, kann dem Arbeitgeber eine Beteiligung an der VAE auf die obligatorische Berufsbildungsabgabe angerechnet werden. Die VAE stellt ein individuelles Recht dar, wobei einige Arbeitgeber ibre Mitarbeiter bei seiner Venvirklicbung begleiten.

Bei der Analyse der französischen Erfahrungen mit der $V A E$ werden die Ambivalenzen sichtbar, die es noch zu beseitigen gilt: In semantischer Hinsicht kann $V A E$ zwei Entwicklungsschemata bezeichnen:

- die Konzeption von ,Diplom' über die Erstausbildung hinweg auf die gesamte Lebensspanne ausweiten oder:

- Möglichkeiten der ,kleinstufigen' Validienung der im Laufe eines, lernenden' Lebens erworbenen Detailkompetenzen bieten.

Tatsächlich entspricht die französische $V A E$, trotz aller Diskurse über lernende Unternehmen, eher dem ersten Schema und nicht dem zweiten. ${ }^{45}$ Ihr Ziel ist es vor allem, das Diplom-Monopol der Erstausbildung zu zerstören und nicht die Kapitalisierung der kleinen individuellen Kompetenzfortschritte im Alltag zu ermöglichen; obwohl gerade hier die Notwendigkeit und das Bedürfnis der Individuen beheimatet ist.

44 RNCP: Repertoire des qualifications professionnelles. Nationales Repertoire beruflicher Qualifikationen.

45 Im Durchschnitt erfordert eine VAE ein Jahr, viel Textarbeit und einige hundert Euro. Siehe beispielsweise Labruyere (2006). 


\section{Das Lebenslange Lemen wird nicht existieren können, solange es kulturell unsicbtbar bleibt}

Mit dem e-learning entdeckt das Lebenslange Lemen die Hauptingredienzien jener pädagogischen Träume, die von den großen Utopisten geschmiedet wurden... Aber um von dieser Utopie zur allgemeinen Verwirklichung zu gelangen, ist die massive Einbeziehung der Bürger selbst sowie die der sozialen Partner im Lebenslangen Lenen unabdingbar. Eine erste Priorität liegt in der Verhandlung klarer Regeln für die Mitwirkung und Verpflichtungen der beteiligten Individuen. Jedoch selbst mit solchen Regeln investieren die Bürger nur, wenn ein überzeugender Motor, nämlich der des sozialen Aufstieges, sie antreibt. Diese Gelegenheit des sozialen Aufstiegs muss erklärt und kommuniziert werden.

Um dies zu tun, muss ein dreifacher kultureller Hebel angesetzt werden. Es geht darum, für alle das Lebenslange Lernen in seiner Rolle als sozialer Fahrstuhl offenkundig zu machen. Des Weiteren muss seine Notwendigkeit hervorgehoben werden, die darin besteht, Räume für Selbstentfaltung und für eine Überwindung der elementaren Kluft zwischen erduldeten Anstrengungen und gewählter Zerstreuung. Diese Aufgabe muss mit der Entwicklung einer Kultur lebenslanger Orientierung einhergehen. Um ein solches Ziel zu erreichen, müssen sowohl die Instrumente der ,Lernermunterung' - wie beispielsweise die VAE - als auch die Informations- und Beratungseinrichtungen - wie die Cité des métiers z. B. - vermehrt werden.

Das Lebenslange Lemen ist auch heute noch lediglich ein mehrdeutiges Agglomerat: Es entspricht weder einem Ort, noch einem greifbaren sozialen Kontext. Es kann sich weder in einem spezifischen Ritus noch in einer identifizierten Zeit wieder erkennen und bleibt auf diese Weise durch traditionelle schulische Vorstellungen geprägt: eher ein Phänomen der Bändigung, der Dressur bzw. der Unterwerfung unter das Wissen als ein Medium des Empowerments und der Emanzipation. Sich für das Lebenslange Lernen einzusetzen heißt in erster Linie seine Sichtbarkeit bzw. Identifizierung voranzutreiben. Aus diesem Grund müssen die Zugänge zum e-learning, Bildungswege und Instrumente wie die $V A E$, das $D I F$ und die Kompetenzbilanzierung einfacher und transparenter werden. Dabei ist es erforderlich, dass all diese Komponenten zusammenwirken, um eine erkennbare Landschaft zu gestalten. In diesem Sinne sind ,Schaufenster' wie die Cité des métiers unentbehrlich, da das Lebenslange Lernen andernfalls lediglich den Formatierungsinteressen der Produktivkräfte folgen, nicht aber der Entfaltung des Menschen dienen würde. 


\section{Zusammenfassung}

Das Lebenslange Lernen, eine humanistische und pragmatische Erfindung, ist auf dem Weg, seine technische Reife zu etlangen. Jedoch ist die Situation auf dem sozioökonomischen Gebiet vor allem durch eine Paradoxie gekennzeichnet: Nach wie vor wird der soziale Status hauptsächlich durch die Erstausbildung determiniert, und überdies bewegt sich Weiterbildung immer auf die bereits qualifizierten Personengruppen zu. Was wäre, wenn die Verbreitung des Lebenslangen Lernens eine soziokulturelle Revolution in sich birgt, die noch aussteht? Oder wenn es die Vorstellungen von Bildung und bildenden Akten selbst sind, die verändert werden müssen, indem auf den Diplombegriff, auf die Spaltung von Zeit oder auf die Idee der lebenslangen Orientierung Einfluss genommen wird?

\section{Résumé}

La formation tout au long de la vie invention humaniste et pragmatique est en train d'arriver à maturité technique. Mais, sur le plan socio-économique, la situation est surtout paradoxale : c'est toujours principalement le niveau de formation initiale qui détermine le niveau social et de plus la formation continue va toujours aux plus qualifiés. Et si la généralisation de la formation tout au long de la vie sous entendait une révolution socioculturelle qui n'a pas encore eu lieu? Et si c'était la représentation de la formation et des actes formatifs qu'il fallait changer en agissant sur la notion de diplôme, sur le clivage des temps ou sur l'idée d'orientation tout au long de la vie?

\section{Literatur}

BELLIER, S. (2001): Le e-learning, pédagogie, contenus, modalités, acteurs. Paris, Éditions Liaisons et Gégos.

Berton, F.; CorreiA, M.; Lespessarles, C. \& MAILlebours, M. (2004): Initiative individuelle et formation, Contributions de la recherche, état des pratiques et étude bibliographique. Paris, L'Harmattan.

BERTON, F. \& CORREIA, M. (2004): Une initiative individuelle de formation entre idéal méritocratique, philosophie humaniste et injonction sociale. In: Berton, F.; Correia, M.; Lespessailes, C. \& Maillebouis, M. (2004): Initiative individuelle et formation, Contributions de la recherche, état des pratiques et étude bibliographique. Paris, L'Harmattan.

http://www.lettre-insertion.fr/documents/correia.pdf 
BERTON, F. (2001): La demande individuelle de formation en cours de vie active et ses particularités institutionnelles françaises. CNAM, Cahier du Lasmas 01-1; http://lasmas.iresco.fr/cahiedoc/c011_Berton.pdf

BONAÏTI, C.; FleURET, A.; POMMIER, P. \& ZOMORA, P. (2006): Pourquoi les moins qualifiés se forment-ils moins? In: DARES, $\mathrm{n}^{\circ} 116$. http://www.travail.gouv.fr/IMG/pdf/DE116_zamora_version_2.pdf

BULLETIN DES BIBLIOTHÈQUES DE FRANCE (2002): Bibliothèque permanente. $\mathrm{N}^{\circ} 3$. http://bbf.enssib.fr/sdx/BBF/frontoffice/2002/03/sommaire.xsp?\#

CARRÉ, P. (2005): L'apprenance, vers un nouveau rapport au savoir. Paris, Dunod.

CARRÉ, P. \& MOISAN, A. (2002): La formation autodirigée. Paris, L'Harmattan.

CARRÉ, P.; MOISAN, A.; POISSON, D. (1997): L'autoformation: psychopédagogie, ingénierie, sociologie, PUF.

CAZES, G. \& POTIER, F. (2002): Un système de vacancier français. In: Viard, J. (dir.) La France des Temps libres et des vacances. Éditions de l'aube / DATAR. Zitiert in: D. Méda (2003): Les nouveaux rapports au temps. In: Dossiers d'études Allocations Familiales, $n^{\circ} 41$.

http://www.caf.fr/web/WebCnaf.nsf/090ba6646193ccc8c125684f005898f3/1d4 e46e4ca5eafd9c1256e300050ce77/\$FILE/Dossier\%2041-

$\% 20$ Univesite $\% \mathrm{C} 3 \% \mathrm{~A} 9 \mathrm{t} \% \mathrm{C} 3 \% \mathrm{~A} 9 . \mathrm{doc}$

CÉREQ BREF (2001): $\mathrm{n}^{\circ}$ 172, février 2001.

CORBIN, A. (1995): L'avènement des loisirs, 1850 - 1960, Aubier.

CREDOC-ENQUÊTE (2002): Conditions de vie et Aspirations des Français.

DARES (2001): Premières informations, premières synthèses. $\mathrm{N}^{\circ} 09-1$.

DEMEULIER, J. (2005): Intervention de cloture au congrès des CEMÉA, Amiens. http://www.cemea.asso.fr/congres/article.php3?id_article $=422$

DOSSIER BIBLIOTHÈQUES ET ÉDUCATION PERMANENTE (2002): Bulletin des Bibliothèque de France, $n^{\circ} 3$

http://bbf.enssib.fr/sdx/BBF/frontoffice/2002/03/sommaire.xsp?\#

DrEvET, D. \& MONOD, A. (1999): Usager acteur... la Cité des métiers. In: Actualité de la formation permanente, $\mathrm{n}^{\circ} 158$.

Drevet, D.; Las Vergnas O. \& ProKhorofF, C. (1996): La culture scientifique et technique face aux fractures sociales: la cité des métiers à La Villette. In: Alliage $n^{\circ} 29-30$, Nice.

DubAR, C. \& GADÉA, C. (1999): La promotion sociale en France. Presses universitaires du Septentrion, coll. Sociologie.

DumAzeDIER, J. (2002): Penser l'autoformation: société d'aujourd'hui et pratiques d'autoformation, Chronique sociale.

DUMAZEDIER, J. (1988): Révolution culturelle du temps libre, Méridiens Klincksieck.

Dumazedier, J. (1962): Vers une civilisation du Loisir. Paris, Seuil.

INSEE (2002): L'évolution des temps sociaux, Economie et statistique $\mathbf{n}^{\circ} 352-353$, INSEE.

http://www.webcommerce.insee.fr/FichesComm/ECO352/ECO352_c1.htm

LABRUYERE, C. (2006): La VAE quels candidats pour quels diplômes?, Bref Cereq, $\mathrm{n}^{\circ} 230$, Marseille, http://www.cereq.fr/pdf/b230.pdf\#search=\%22etude $\% 20 \mathrm{VAE} \% 22$ 
LAS VERGNAS, O. (2006): Nos sociétés prendront-elles le gai savoir au sérieux? In: Revue Savoirs, $\mathrm{n}^{\circ} 10$, Université Paris X.

LAS Vergnas, O. (2005): Des cités des métiers et de la santé à l'empowerment. In: Desclaux, B. \& Guerrier, R (dir.): Actes du Colloque international "Orientation passé, présent, avenir", L'orientation scolaire et professionnelle. Sonderausgabe, Band 3, INETOP CNAM, Paris.

LAS VERGNAS, O. (1998): la Cité des métiers au service de ses usagers, In: Connaître les publics, savoir pour agir. In: Koenig, M.-H. (dir.): Institut de formation des bibliothécaires, Villeurbanne.

MARESCA, B. ET AL. (2004): Occupation du temps libre, une norme de consommation inégalement partagée, CREDOC, cahier de recherche $n^{\circ} 210$. http://www.credoc.fr/pdf/Rech/C210.pdf

PINEAU, G. (1995): L'autoformation en chantiers, Éducation permanente, n ${ }^{\circ} 122$.

PODEVIN, G. (1999): formation: promotion sociale et professionnelle: un lien démocratique rompu. In: Dubar, C. \& Gadéa, Ch. (dir.): La promotion sociale en France, Presses universitaires du Septentrion, coll. Sociologie.

POLIAK, C. (1992): La vocation d'autodidacte. L'Harmattan.

REVUE SCIENCES HUMAINES (2003): Former, se former, se transformer. Sonderausgabe, Mars/Avril/Mai, $n^{\circ} 40$.

RIVERIN, D. \& SIMARD, Y. (2003): Au-delà du lien travail-loisir, la citoyenneté. Revue Francophone Internationale de Carriérologie, Vol $9 n^{\circ} 2$

http://www.carrierologie.uqam.ca/volume09_1-2/13_riverin/index.html

VERRIER, C. (1999): Autodidaxie et autodidactes, l'infini des possibles. Anthropos.

VIARD, J. (2002): Le sacre du temps libre: la société des 35 heures. Editions de l'aube.

YONNET, P. (1999): Travail, loisirs, temps libres et lien social, Gallimard.

\section{Internetquellen}

http://www.cereq.fr/cereq/fc2000/Default.htm\#Présentation http://www.plan.gouv.fr/organisation/sas/PMQ/seance7-atel3.htm http://www.reseaucitesdesmetiers.org http://www.cemea.asso.fr/congres/

\section{Biograpbische Angaben}

\section{Dr. Olivier Las Vergnas}

Olivier Las Vergnas ist Astrophysiker und Spezialist im Bereich der Systeme beruflicher Bildung. Er hat 1993 die Gründung der Cité des métiers betrieben, ein für alle Personengruppen offener und zugänglicher Raum der Information und Beratung, mitten in der Cité des sciences et de l'industrie von La Vilette in 
Paris. Er leitet diesen Verbund, in dem 14 Organisationen zusammenarbeiten, seit seiner Entstehung und ist Generalsekretär des Internationalen Netzwerkes der Cité des métiers, das nach dem Pariser Vorbild entstanden ist. Weiterhin wirkt er mit bei Verbänden, die sich mit der Verbreitung von wissenschaftlichem und technischem Wissen in Frankreich beschäftigen. 
Brought to you by | Université de Lille I - Sciences et Technologies 